\title{
ENFERMEDAD DE LYME EN EL PERÚ. UNA REVISIÓN CLÍNICA Y EPIDEMIOLÓGICA
}

\author{
Jorge Cervantes ${ }^{1, a}$
}

\begin{abstract}
RESUMEN
Esta es una revisión crítica y organizada de la información disponible y actualizada acerca de la enfermedad de Lyme y la infección por Borrelia en el Perú. Varios estudios de serología contra Borrelia burgdorferi, y de casos de enfermedad de Lyme han sido reportados en el Perú en las pasadas dos décadas. Nueva información sugiere la existencia de nuevas especies de Borrelia burgdorferi sensu lato en Sudamérica, y posiblemente en el Perú. Futuros estudios genéticos y microbiológicos en esta parte del continente, no sólo en casos con Western blot indeterminado, sino también en vectores y posibles reservorios, son necesarios para medir la extensión de estas nuevas especies de Borrelia y su implicancia clínica.
\end{abstract}

Palabras clave: Enfermedad de lyme; Borrelia; Perú. (fuente: DeCS BIREME).

\section{LYME DISEASE IN PERU. A CLINICAL AND EPIDEMIOLOGICAL REVIEW}

\begin{abstract}
This is a critical and organized review of all the available and updated information on Lyme disease and Borrelia infection in Peru. Several studies of positive serology to Borrelia burgdorferi and several cases of Lyme disease have been reported in Peru in the last two decades. New information suggests that new species of Borrelia burgdorferi sensu lato do exist in South America, and possibly in Peru. Future genetic and microbiology studies in this part of the continent, not only in cases with an indeterminate Western blot but in vectors and possible reservoirs as well, are necessary to measure the extent and clinical implications of these new Borrelia species
\end{abstract}

Keywords: Lyme disease; Borrelia; Peru (source: MeSH NLM).

\section{LA ENFERMEDAD DE LYME: UN PROBLEMA GLOBAL}

La enfermedad de Lyme es una zoonosis causada por la espiroqueta Borrelia burgdorferi sensu lato (B.b.s.l.), la cual es transmitida por garrapatas ${ }^{(1)}$. La enfermedad fue descrita en los años sesenta por Allen Steere durante un brote epidémico de artritis reumatoidea juvenil en Connecticut (2). Steere, quien había tenido entrenamiento previo en el Centro para el Control de Enfermedades (CDC, por sus siglas en Inglés), sospechaba de la naturaleza infecciosa de la enfermedad, y fue así que se descubrió su asociación con un vector ${ }^{(3)}$. Manifestaciones dérmicas de la enfermedad de Lyme, tales como el erythema migrans (EM), ya habían sido descritas a principios del siglo $X X{ }^{(4)}$. La espiroqueta B.b. fue hallada en el tubo digestivo de garrapatas del género Ixodes por Burgdorfer en $1984{ }^{(5)}$.

El hallazgo de partes del genoma de B.b. en un estudio genómico del «hombre de hielo», una momia hallada en los Alpes con una antigüedad de 5,300 años, sugiere la espiroqueta ha afectado a la humanidad desde hace miles de años ${ }^{(6)}$. Esta especulación, cobra aún más plausibilidad, luego de encontrarse un sinnúmero de tatuajes en la piel de esta momia, en puntos tradicionalmente usados por la terapia de acupuntura ${ }^{(7)}$. La enfermedad de Lyme es, en esencia, una enfermedad inflamatoria multisistémica, donde las manifestaciones clínicas se deben a una respuesta exacerbada del hospedero ${ }^{(8)}$.

Una enfermedad infecciosa emergente se define como aquella que es la provocada por un agente infeccioso recientemente identificado y anteriormente desconocido, capaz de causar problemas de salud pública a nivel local, regional o mundial ${ }^{(9)}$. La enfermedad de Lyme constituye una enfermedad infecciosa emergente de proporciones globales. A pesar de haber sido descrita en Norteamérica, la borreliosis es endémica en muchos países de Europa y Asia ${ }^{(1,10)}$. También ha sido descrita en África del Norte ${ }^{(1,11)}$, Oceanía ${ }^{(12)}$ y Sudamérica ${ }^{(13)}$.

Paul L. Foster School of Medicine, Texas Tech University Health Sciences Center. El Paso, Texas, EE. UU.

Médico cirujano.

Recibido: 26/01/2018 Aprobado: 25/04/2018 En línea: 28/06/2018

Citar como: Cervantes J. Enfermedad de Lyme en el Perú. Una revisión clínica y epidemiológica. Rev Peru Med Exp Salud Publica. 2018;35(2): 292-6. doi:10.17843/rpmesp.2018.352.3418 
En vista de las muchas publicaciones que han estado apareciendo sobre enfermedad de Lyme en esta parte del continente, y debido a la inconsistencia de los hallazgos, una revisión con miras a clarificar los patrones emergentes de la infección por Borrelia en Sudamérica no sólo es justificable sino también oportuno.

La estrategia de búsqueda se realizó en PubMed, así como en buscadores de internet Google y Bing, debido a que algunas revistas Latinoamericanas no están indexadas y no aparecen en búsquedas realizadas a través de PubMed. La estrategia de búsqueda incluyó los términos «Lyme» OR «Lyme disease» OR «Lyme Disease spirochete» OR «enfermedad de Lyme» OR «Borreliosis» OR «Borrelia» OR «Borrelia infection» OR «infección por Borrelia» AND «Latin America» OR «Southamerica» OR «Sudamerica» $O R$ «Argentina» $O R$ «Chile» $O R$ «Colombia» $O R$ «Peru»OR «Bolivia» OR «Brazil» OR «Ecuador”. La búsqueda se llevó a cabo desde la concepción del estudio de revisión hasta enero del 2018. No se aplicaron restricciones de lenguaje en dichas búsquedas.

\section{BORRELIA Y VECTORES}

Las especies del género Borrelia asociadas con la enfermedad de Lyme están agrupadas en un complejo de genoespecies llamadas B.b.s.l., que incluye a la Borrelia burgdorferi sensu stricto (B.b.s.s) ${ }^{(14,15)}$. Existe cierta especificidad entre Borrelia y garrapata ${ }^{(16-18)}$, de modo que las diferentes especies de Borrelia utilizan diferentes vectores para su transmisión en distintas áreas geográficas ${ }^{(1,11)}$. Más aún, diferentes especies de Borrelia están asociadas con diferentes presentaciones clínicas de la enfermedad (19).

Borrelia existe en la naturaleza en un ciclo enzoótico, en garrapatas, mayormente del género Ixodes ${ }^{(3)}$. En Norteamérica, B.b. es transmitida mayormente por Ixodes scapularis e Ixodes pacificus ${ }^{(20,21)}$, mientras que en Europa y Asia Ixodes ricinus e Ixodes persulcatus, respectivamente, son mayormente utilizados ${ }^{(15)}$. Varias especies del género Ixodes (I. affinis, I. pararicinus, I. ricinus, e I. boliviensis), así como del género Amblyomma han sido descritas en el Perú (22-26). Se desconoce si estas especies en el area geográfica peruana están infectadas con Borrelia, como se ha demostrado en Argentina por ejemplo, donde una fracción de I.pararicinus están infectada con B.b.s.l. (27) . La especie Ixodes pararicinus se encuentra distribuída en todo Sudamérica incluyendo el Perú ${ }^{(28,29)}$. Ácaros pertenecientes a la especie Trombicula autumnalis son endémicos en la selva peruana ${ }^{(30)}$ y en Argentina, donde se les conoce como «isangos» o «bichos colorados», respectivamente. Es posible que este sirva como vector de B.b., como se ha demostrado en otras partes del mundo ${ }^{(31)}$.

Se cree que estas garrapatas fueron introducidas en el nuevo mundo por animales ungulados como el venado.
Se han encontrado larvas y ninfas de $I$. pararicinus en roedores y aves de esta región (29). Garrapatas de la familia Ixodidae, pero pertenecientes a otros géneros, tales como Haemaphysalis y Amblyomma también han sido encontradas en aves sudamericanas. El ciclo zoonótico de Borrelia involucra reservorios en la naturaleza tales como el roedor Peromyscus leucopus y el venado Odocoileus virginianus. En Sudamérica, varias especies de aves, roedores y lagomorfos tales como la liebre se han propuesto como reservorios ${ }^{(32,33)}$.

\section{ENFERMEDAD DE LYME EN EL PERÚ}

Un reporte de vigilancia sindrómica de la Dirección General de Epidemiologia del Ministerio de Salud, menciona a la enfermedad de Lyme en la lista de patógenos y enfermedades de identificación reciente para

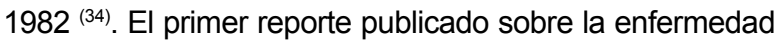
de Lyme en el Perú ocurrió en 1991, donde un estudio retrospectivo en suero de 216 agricultores identificó cuatro (2\%) muestras positivas para anticuerpos contra B.b. ${ }^{(23)}$. Esta seroprevalencia es similar a la que luego se reportara en agricultores de Colombia (4,6\%) ${ }^{(35)}$. Un estudio serológico posterior en Piura y Amazonas, en poblaciones que se dedicaban principalmente a la agricultura, encontró anticuerpos contra B.b. en $10 \%$ de un total de 232 individuos sanos ${ }^{(22)}$. Una cifra un poco más elevada se observó casi al mismo tiempo en población rural sana de Tarija, Bolivia (3\%) ${ }^{(36)}$. Es importante destacar que estos estudios también detectaron presencia de garrapatas de los géneros Ixodes, Amblyomma, Rhipicephalus, Anocentor, y Boophilus en Piura y Amazonas ${ }^{(22)}$, mientras que en Bolivia las garrapatas pertenecían al género Dermacentor ${ }^{(36)}$.

Entre 1992 y 1999 se reportaron seis casos de enfermedad de Lyme en el Perú ( ${ }^{37}$ ). Todos estos casos tenían serología positiva por ELISA, y uno por Western blot. Uno de estos casos presentaba erythema migrans (EM) con visualización directa de espiroquetas por microscopía. A esto hay que sumar el reporte de un caso de EM con serología positiva por ELISA, cuyos títulos fueron disminuyendo luego de la terapia antibiótica ${ }^{(38)}$. Un estudio en Brasil detectó presencia de espiroquetas en cinco de un total de 22 pacientes con EM (39), así como en pacientes con síndrome similar a enfermedad de Lyme ${ }^{(40)}$.

El síndrome de Parry-Romberg (SPR) es una entidad rara, caracterizada por atrofia hemifacial progresiva ${ }^{(41)}$. Varios casos de SPR han presentado presencia de anticuerpos IgM contra B.b., e inclusive un cultivo positivo para B.b. ${ }^{(42-44)}$. Dos casos de SPR con serología positiva a IgM han sido reportados en el Perú ${ }^{(42)}$.

A todos estos casos, hay que sumar un reporte de serología positiva en perros examinados por decaimiento general (45) (Tabla 1). 
Tabla 1. Reporte de casos de enfermedad de Lyme en el Perú

\begin{tabular}{|c|c|c|c|c|c|}
\hline Autor & Año & Localidad & Estudio & Método & Hallazgo \\
\hline Need y Escamilla ${ }^{(23)}$ & 1991 & Desconocida & Retrospectivo & ELISA & $\begin{array}{l}\text { De un total de sueros de } 216 \text { sujetos, cuatro } \\
(2 \%) \text { fueron positivos para anticuerpos contra } \\
\text { Bb. }\end{array}$ \\
\hline Romero ${ }^{(38)}$ & 1996 & Lima & $\begin{array}{l}\text { Reporte de un } \\
\text { caso }\end{array}$ & ELISA & $\begin{array}{l}\text { Un caso de erythema migrans (EM) con } \\
\text { IgM positiva }\end{array}$ \\
\hline Castillo Rivadeneyra ${ }^{(37)}$ & 1999 & Lima & $\begin{array}{l}\text { Reporte de seis } \\
\text { caso }\end{array}$ & $\begin{array}{l}\text { ELISA / } \\
\text { microscopia }\end{array}$ & $\begin{array}{l}\text { Seis casos, con serología positiva. Uno } \\
\text { con erythema migrans (EM) y visualización } \\
\text { microscópica de espiroquetas. }\end{array}$ \\
\hline Glenny, M. et al. (22) & 2004 & Piura y Amazonas & Serológico & ELISA & $\begin{array}{l}23 \text { de } 232(10 \%) \text { sueros de individuos sanos } \\
\text { presentaron anticuerpos con B.b. }\end{array}$ \\
\hline Galarza ${ }^{(42)}$ & 2006 & Cusco & $\begin{array}{l}\text { Reporte de } \\
\text { casos }\end{array}$ & ELISA & $\begin{array}{l}\text { Dos pacientes con síndrome de Parry- } \\
\text { Romberg con serología positiva (IgM) para } \\
\text { B.b. }\end{array}$ \\
\hline Rubio, A.M. et al. ${ }^{(45)}$ & 2011 & Lima & $\begin{array}{l}\text { Serológico / } \\
\text { Perros }\end{array}$ & ELISA & $\begin{array}{l}\text { Serología positiva contra B.b. en dos perrros } \\
\text { examinados por decaimiento general. Sin } \\
\text { historia de garrapatas. }\end{array}$ \\
\hline
\end{tabular}

\section{LIMITACIONES DE LA EVALUACIÓN SEROLOGICA}

A pesar de que los resultados positivos de serología no constituyen evidencia concluyente de la presencia de borreliosis de Lyme en Sudamérica, representan evidencia indirecta de exposición a Borrelia. La especificidad de pruebas diagnósticas tendría un impacto en la detección de cepas «locales» de Sudamérica, que serían antigénicamente distintas, pero igualmente importante en la posibilidad de reacción cruzada con otras especies de Borrelia, tales como B. turicatae y B. parkeri, causantes de la fiebre recurrente ${ }^{(46)}$.

En algunos casos no es posible determinar si los resultados de serología corresponden a lgM o lgG. Falsos positivos para IgM contra Borrelia han sido reportados en pacientes con factor reumatoideo $\operatorname{lgM}{ }^{(47)}$. Reacciones cruzadas con otras espiroquetas como Leptospira, Treponema pallidum, Treponema phagedenis ${ }^{(48)}$, y con el virus de inmunodeficiencia humana, pueden ser distinguidas a través del western-blot ${ }^{(49)}$.

Dos meta-análisis de estudios serológicos de enfermedad de Lyme entre 1995 y 2016 encontraron que la media de sensibilidad para todas las pruebas era de $59,5 \%{ }^{(50)}$ y de $57,6 \%{ }^{(51)}$. El western-blot presentó mayor sensibilidad que el ELISA, cuya sensibilidad estuvo alrededor del $53 \%{ }^{(50)}$. Ya que lo que se miden con estas pruebas es el nivel de anticuerpos del hospedero, no es sorpresa que la sensibilidad fuese muy baja en la enfermedad aguda, comparado con aquella en convalescencia ${ }^{(50)}$, donde puede llegar a ser mayor del $99 \%{ }^{(51)}$. Muchos de los estudios serológicos en los casos reportados no dan detalles del tipo de prueba diagnóstica utilizada, o si lo hacen, no mencionan el nombre del kit de ELISA o Western-blot. Esto es importante, ya que existe una gran variedad en la sensibilidad y especificidad de los diferentes kits comerciales de ELISA y de Westernblot ${ }^{(51)}$. Estos valores suelen mejorar si se realiza la serología combinando un ELISA con una prueba de Western-blot; sin embargo, esto también está sujeto a la marca de kit utilizada ${ }^{(51)}$. La prueba serológica sólo puede ser útil dentro de un contexto clínico ${ }^{(52)}$, y su utilización en sujetos con síntomas subjetivos inespecíficos no es recomendable, ya que disminuye el valor predictivo positivo ${ }^{(53)}$.

\section{NUEVAS ESPECIES DE BORRELIA EN SUDAMÉRICA}

Varios reportes en Sudamérica han mostrado que, a pesar de mostrar un resultado de ELISA positivo, muchos casos de presunta enfermedad de Lyme no logran alcanzar el criterio de Western blot para ser considerados positivos ${ }^{(54-57)}$. Esto llevó a sugerir la hipótesis de la existencia de una nueva cepa local de B.b.s.l., antigénicamente distinta de la cepa americana B31, que es usualmente utilizada como fuente antigénica para los exámenes de diagnóstico ${ }^{(50)}$. Esta teoría cobra aún más solidez al evidenciarse la presencia de genoma de B.b. en Brasil por reacción en cadena de la polimerasa (PCR) utilizando una batería de primers (cebadores) ${ }^{(13)}$. El análisis filogenético ha revelado que en algunos casos en Brasil se trata de B.garinii, B.b.s.s. ${ }^{(58)}$ o B.b.s.l. haplotipo Pampa ${ }^{(59)}$.

En Uruguay y Chile, nuevas especies del género Borrelia han sido reportadas. Las nuevas especies detectadas en Uruguay provienen de garrapatas recolectadas de venados, ganado, y vegetación local, y parecen estar filogenéticamente cercanas a $B$. bissettii, y a $B$. americana ${ }^{(60)}$. En Chile, la nueva especie ha sido denominada Borrelia chilensis, y se ha encontrado en garrapatas de la especie Ixodes stilesi, en roedores, venados, y vegetación de esa región ${ }^{(61-63)}$, así como en otras garrapatas del género Ixodes en la Patagonia ${ }^{(64)}$, y el Noroeste de Argentina ${ }^{(27,65)}$. 


\section{CONCLUSIONES}

La información recolectada indica la presencia de los principales elementos para esta zoonosis en Sudamérica, a saber, reservorios como venados, ganado, roedores y aves, vectores del género /xodes. El ser humano es un hospedero accidental del ciclo zoonótico mantenido en la naturaleza. Los hallazgos de reportes de casos clínicos compatibles con infección por B.b., con o sin confirmación serológica, aunado a la presencia de especies de garrapatas en las que se ha hallado infección por nuevas especies de Borrelia, hace sospechar que estas podrían ser responsables por los casos de borreliosis en nuestro país. La infección por estas nuevas especies de Borrelia podría además, identificar casos de fiebre de origen desconocido en nuestro país. Futuros estudios genéticos y microbiológicos en esta parte del continente, no sólo en casos con Western blot indeterminado, sino también en vectores y posibles reservorios, son necesarios para medir la extensión de estas nuevas especies de Borrelia y su implicancia clínica.

Fuentes de financiamiento: Autofinanciado.

Conflictos de interés: El autor declara no tener conflictos de interes en la publicación de este artículo.

\section{REFERENCIAS BIBLIOGRÁFICAS}

1. Schotthoefer AM, Frost HM. Ecology and Epidemiology of Lyme Borreliosis. Clin Lab Med. 2015;35(4):723-43.

2. Borchers AT, Keen CL, Huntley AC, Gershwin ME. Lyme disease: a rigorous review of diagnostic criteria and treatment. J Autoimmun. 2015;57:82-115.

3. Steere AC. Lyme disease: a growing threat to urban populations. Proc Natl Acad Sci US A. 1994;91(7):2378-83.

4. Steere AC. Lyme borreliosis in 2005,30 years after initial observations in Lyme Connecticut. Wien Klin Wochenschr. 2006;118(21-22):625-33.

5. Burgdorfer W.Discovery of the Lyme disease spirochete and its relation to tick vectors. Yale J Biol Med. 1984;57(4):515-20.

6. Keller A, Graefen A, Ball M, Matzas M, Boisguerin V, Maixner F, et al. New insights into the Tyrolean Iceman's origin and phenotype as inferred by wholegenome sequencing. Nat Commun. 2012;28(3):698.

7. Deter-Wolf A, Robitaille B, Krutak L, Galliot S. The world's oldest tattoos. J Archaeol Sci-Rep. 2016;5:19-24.

8. Cervantes J. Doctor says you are cured, but you still feel the pain. Borrelia DNA persistence in Lyme disease. Microbes Infect. 2017;19(9-10):459-63.

9. Oromi Durich J. Enfermedades emergentes y reemergentes: algunas causas y ejemplos. Med Integr . 2000;36(3):79-82.

10. Mead PS. Epidemiology of Lyme disease. Infect Dis Clin North Am. 2015;29(2):187-210.

11. Stone BL, Tourand Y, Brissette CA. Brave New Worlds: The Expanding Universe of Lyme Disease. Vector Borne Zoonotic Dis. 2017;17(9):619-29.

12. Collignon PJ, Lum GD, Robson JM. Does Lyme disease exist in Australia? Med J Aust. 2016;205(9):413-7.
13. Mantovani E, Marangoni RG, Gauditano G, Bonoldi VL, Yoshinari NH. Amplification of the flgE gene provides evidence for the existence of a Brazilian borreliosis. Rev Inst Med Trop Sao Paulo. 2012;54(3):153-7.

14. Margos G, Vollmer SA, Ogden NH, Fish D. Population genetics, taxonomy, phylogeny and evolution of Borrelia burgdorferi sensu lato. Infect Genet Evol. 2011;11(7):1545-63.

15. Mechai S, Margos G, Feil EJ, Barairo N, Lindsay LR, Michel P, et al. Evidence for Host-Genotype Associations of Borrelia burgdorferi Sensu Stricto. PLoS One. 2016;11(2):e0149345.

16. Masuzawa $T$. Terrestrial distribution of the Lyme borreliosis agent Borrelia burgdorferi sensu lato in East Asia. Jpn J Infect Dis. 2004;57(6):229-35.

17. Liu S, Yuan C, Cui YF, Li BX, Wu LJ, Liu Y. Investigation of Borrelia spp. in ticks (Acari: Ixodidae) at the border crossings between China and Russia in Heilongjiang Province, China. Asian Pac J Trop Med. 2012;5(6):459-64.

18. Ni XB, Jia N, Jiang BG, Sun T, Zheng YC, Huo QB, et al. Lyme borreliosis caused by diverse genospecies of Borrelia burgdorferi sensu lato in northeastern China. Clin Microbiol Infect. 2014;20(8):808-14.

19. Kurtenbach K, Hanincova K, Tsao JI, Margos G, Fish D, Ogden NH. Fundamental processes in the evolutionary ecology of Lyme borreliosis. Nat Rev Microbiol. 2006;4(9):660-9.

20. Oliver JH Jr, Chandler FW Jr, Luttrell MP, James AM, Stallknecht DE, McGuire $\mathrm{BS}$, et al. Isolation and transmission of the Lyme disease spirochete from the southeastern United States. Proc Natl Acad Sci U S A. 1993;90(15):7371-5.
21. Khatchikian CE, Prusinski M, Stone M Backenson PB, Wang IN, Levy MZ, et al. Geographical and environmental factors driving the increase in the Lyme disease vector Ixodes scapularis. Ecosphere. 2012;3(10).

22. Glenny M, Mendoza L, Falconi E. Detección de anticuerpos contra Borrelia burgdorferi e identificación de garrapatas ixodidas en Piura y Amazonas, Perú. Rev Peru Med Exp Salud Publica. 2004;20(1):23-7.

23. Need JT,Escamilla J.Lyme disease in South America? J Infect Dis. 1991;163(3):681-2.

24. Mendoza-Uribe L, Chávez-Chorocco J. Ampliacion geografica de siete especies de Amblyomma (Acari: Ixodidae) y primer reporte de A. oblongoguttatum Koch, 1844 para Peru. Rev Peru Ent. 2004;44(1):66-72.

25. Escalante JA, Mollinedo L. Ocho artropodos de interes veterinario en el Cusco Rev Per Entomol. 1989;31:46-7.

26. Blair PJ, Jiang J, Schoeler GB, Moron C, Anaya E, Cespedes M, et al. Characterization of spotted fever group rickettsiae in flea and tick specimens from northern Peru. J Clin Microbiol. 2004;42(11):4961-7.

27. Nava S, Barbieri AM, Maya L, Colina R, Mangold AJ, Labruna MB, et al. Borrelia infection in Ixodes pararicinus ticks (Acari: Ixodidae) from northwestern Argentina. Acta Trop. 2014;139:1-4.

28. Venzal JM, Estrada-Pena A, Barros-Battesti DM, Onofrio VC, Beldomenico PM. Ixodes (Ixodes) pararicinus Keirans \& Clifford, 1985 (Acari: Ixodidae): description of the immature stages, distribution, hosts and medical/veterinary importance. Syst Parasitol. 2005;60(3):225-34.

29. Venzal JM, Felix ML, Olmos A, Mangold AJ, Guglielmone AA. A collection of ticks (Ixodidae) from wild birds in Uruguay. Exp Appl Acarol. 2005;36(4):325-31. 
30. Beltran M, Valdivia, C, Ponce-Ramirez, R, Chambergo, M. Trombicula autumnalis (isangos) en un jardín de niños de la selva peruana. Rev Peru Med Exp Salud Publica. 2009;26(1):58-60.

31. Kampen H, Scholer A, Metzen M, Oehme R, Hartelt K, KimmigP, et al.Neotrombicula autumnalis (Acari, Trombiculidae) as a vector for Borrelia burgdorferi sensu lato? Exp Appl Acarol. 2004;33(1-2):93-102.

32. Osorio AG. Búsqueda de la espiroqueta Borrelia burgdorferi sensu lato mediante PCR en garrapatas ixoideas chilenas silvestres. Rev Med Chile. 2001;129(3):270-6.

33. González-Acuña D, Venzal JM, Guglielmone AA. Nuevos hospedadores y localidades de Ixodes sigelos e Ixodes auritulus (Acari: Ixodidae) en Chile. Gayana. 2004;68:108-11.

34. Tejada Vasquez E. Vigilancia Sindromica. Ministerio de Salud. Direccion General de Epidemiologia; 2014 Octubre, 2014.

35. Miranda J, Mattar S, Perdomo K, Palencia L. [Seroprevalence of Lyme borreliosis in workers from Cordoba, Colombia]. Rev Salud Publica (Bogota). 2009;11(3):480-9.

36. Briancon C. Enfermedad de Lyme en areas presuntamente endemicas del departamento de Tarija-Bolivia. Rev Inst Med Sucre. 2005;126:33-9.

37. Castillo Rivadeneyra WaCF. Borreliosis de Lyme en el Perú. Revista de Farmacologia Terapeutica. 1999;6(1-2):72-4.

38. Romero OG, C; Rueda, M. Eritema Cronico Migrans. Dermatologia Peruana. 1996;6(1-2).

39. Talhari S, de Souza Santos MN, Talhari C, de Lima Ferreira LC, Silva RM, Jr. Zelger B, et al. Borrelia Burgdorferi «sensu lato» in Brazil: Occurrence confirmed by immunohistochemistry and focus floating microscopy. Acta Trop. 2010;115(3):200-4.

40. Mantovani E, Costa IP, Gauditano G, Bonoldi VL, Higuchi ML, Yoshinari NH. Description of Lyme disease-like syndrome in Brazil. Is it a new tick borne disease or Lyme disease variation? Braz J Med Biol Res. 2007;40(4):443-56.

41. Castaneda-Reyna MA, and GalarzaManyari, C. Sindrome de Parry-Romberg asociado a epilepsia refractaria, atrofia de la duramadre y leucoencefalopatia quistica cerebral. Rev Neurol. 2003;37:941-5.

42. Galarza C, Gutierrez E, Ramos W, Macetas R, Mendoza M, Gomez A, et al. Síndrome de Parry Romberg. Dermatol Peru. 2006;16(2):151-4.

43. Sahin MT, Baris S, Karaman A. ParryRomberg syndrome: a possible association with borreliosis. J Eur Acad Dermatol Venereol. 2004;18(2):204-7.
44. Baskan EB, Kacar SD, Turan A, Saricaoglu H, Tunali S, Adim SB. Parry-Romberg syndrome associated with borreliosis: could photochemotherapy halt the progression of the disease? Photodermatol Photoimmunol Photomed. 2006;22(5):259-61.

45. Rubio AM, Salas EA, Gomez G. Presence of Antibodies against Borrelia burgdorferi and Anaplasma so. in dogs in Lima, Peru. Rev Inv Vet Peru. 2011;22(3):233-8.

46. Ciceroni L, Bartoloni A, Guglielmetti P, Paradisi F, Barahona HG, Roselli M, et al. Prevalence of antibodies to Borrelia burgdorferi, Borrelia parkeri and Borrelia turicatae in human settlements of the Cordillera Province, Bolivia. J Trop Med Hyg. 1994;97(1):13-7.

47. Barka NE, Agopian MS, Peter JB. Falsepositive IgM antibodies to Borrelia burgdorferi in indirect ELISA as a result of IgM rheumatoid factor. J Infect Dis. 1990;161(6): 1312.

48. Magnarelli LA, Miller JN, Anderson JF, Riviere GR. Cross-reactivity of nonspecific treponemal antibody in serologic tests for Lyme disease. J Clin Microbiol. 1990;28(6):1276-9.

49. Raoult D, Hechemy KE, Baranton G. Cross-reaction with Borrelia burgdorferi antigen of sera from patients with human immunodeficiency virus infection, syphilis, and leptospirosis. J Clin Microbiol. 1989;27(10):2152-5.

50. Cook MJ, Puri BK. Commercial test kits for detection of Lyme borreliosis: a metaanalysis of test accuracy. Int J Gen Med. 2016;9:427-40.

51. Waddell LA, Greig J, Mascarenhas M, Harding S, Lindsay R, Ogden N. The Accuracy ofDiagnostic Tests for LymeDisease in Humans, A Systematic Review and Meta-Analysis of North American Research. PLoS One. 2016;11(12):e0168613.

52. Lienhard R. Lyme serology: what's the deal?. Rev Med Suisse. 2015;11(489):1830-4.

53. Dessau RB, van Dam AP, Fingerle V, Gray J, Hovius JW, Hunfeld KP, et al. To test or not to test? Laboratory support for the diagnosis of Lyme borreliosis Author's reply. Clin Microbiol Infect. 2018;24(2):211-2

54. Santos M, Ribeiro-Rodrigues R, Lobo R Talhari S. Antibody reactivity to Borrelia burgdorferi sensu stricto antigens in patients from the Brazilian Amazon region with skin diseases not related to Lyme disease. Int J Dermatol. 2010;49(5):552-6.

55. Neira O, Cerda C, Alvarado MA, Palma $S$, Abumohor P, Wainstein E, et al. Lyme disease in Chile. Prevalence study in selected groups. Rev Med Chil. 1996;124(5):537-44.
56. Palacios R, Osorio LE, Giraldo LE, Torres AJ, Philipp MT, Ochoa MT. Positive IgG Western blot for Borrelia burgdorferi in Colombia. Mem Inst Oswaldo Cruz. 1999;94(4):499-503.

57. Carranza-Tamayo CO, Costa JN, Bastos WM. Lyme disease in the state of Tocantins, Brazil: report of the first cases. Braz J Infect Dis. 2012;16(6):586-9.

58. Goncalves DD, Moura RA, Nunes M, Carreira T, Vidotto O, Freitas JC, et al. Borrelia burgdorferi sensu lato in humans in a rural area of Parana State, Brazil. Braz J Microbiol. 2015;46(2):571-5.

59. Dall'Agnol B, Michel T, Weck B, Souza UA, Webster A, Leal BF, et al. Borrelia burgdorferi sensu lato in Ixodes longiscutatus ticks from Brazilian Pampa. Ticks Tick Borne Dis. 2017;8(6):928-32.

60. Barbieri AM, Venzal JM, Marcili A, Almeida AP, Gonzalez EM, Labruna MB. Borrelia burgdorferi sensu lato infecting ticks of the Ixodes ricinus complex in Uruguay: first report for the Southern Hemisphere. Vector Borne Zoonotic Dis. 2013;13(3):147-53.

61. Ivanova LB, Tomova A, Gonzalez-Acuna D, Murua R, Moreno CX, Hernandez $\mathrm{C}$, et al. Borrelia chilensis, a new member of the Borrelia burgdorferi sensu lato complex that extends the range of this genospecies in the Southern Hemisphere. Environ Microbiol. 2014;16(4):1069-80.

62. Huang W, Ojaimi C, Fallon JT, Travisany $\mathrm{D}$, Maass A, Ivanova L, et al. Genome Sequence of Borrelia chilensis VA1, a South American Member of the Lyme Borreliosis Group. Genome Announc. 2015;3(1).

63. Verdugo C, Jimenez O, Hernandez C, Alvarez P, Espinoza A, Gonzalez-Acuna D. Infection with Borrelia chilensis in Ixodes stilesi ticks collected from Pudu puda deer. Ticks Tick Borne Dis. 2017;8(5):733-40.

64. Sebastian PS, Bottero MN, Carvalho L, Mackenstedt U, Lareschi M, Venzal JM, et al. Borrelia burgdorferi sensu lato in Ixodes cf. neuquenensis and Ixodes sigelos ticks from the Patagonian region of Argentina. Acta Trop. 2016;162:218-21.

65. Saracho Bottero MN, Sebastian PS, Carvalho LA, Claps LG, Mastropaolo M, Mangold AJ, et al. Presence of Borrelia in different populations of Ixodes pararicinus from northwestern Argentina. Ticks Tick Borne Dis. 2017;8(4):488-93.

Correspondencia: Jorge L. Cervantes

Dirección: 5001 El Paso Dr., El Paso, TX 79905, U.S.A.

Teléfono: +1-915-215-4672

Correoelectrónico:Jorge.cervantes@ttubsc.edu 